\title{
Separable Kernel for Image Deblurring
}

\author{
Lu Fang ${ }^{1 *}$, Haifeng $\mathrm{Liu}^{1}$, Feng $\mathrm{Wu}^{1}$, Xiaoyan $\mathrm{Sun}^{2}$, Houqiang $\mathrm{Li}^{1}$ \\ ${ }^{1}$ University of Science and Technology of China, Hefei, China \{fanglu, lemt, fengwu, hqli\}@ustc.edu.cn \\ ${ }^{2}$ Microsoft Research Asia, Beijing, China xysun@microsoft.com
}

\begin{abstract}
In this paper, we deal with the image deblurring problem in a completely new perspective by proposing separable kernel to represent the inherent properties of the camera and scene system. Specifically, we decompose a blur kernel into three individual descriptors (trajectory, intensity and point spread function) so that they can be optimized separately. To demonstrate the advantages, we extract one-pixel-width trajectories of blur kernels and propose a random perturbation algorithm to optimize them but still keeping their continuity. For many cases, where current deblurring approaches fall into local minimum, excellent deblurred results and correct blur kernels can be obtained by individually optimizing the kernel trajectories. Our work strongly suggests that more constraints and priors should be introduced to blur kernels in solving the deblurring problem because blur kernels have lower dimensions than images.
\end{abstract}

\section{Introduction}

The blur effects caused by camera shake and object motion in scenes occur frequently in photography, producing disappointing blurry images with inevitable information loss, which becomes one of the most common reasons for discarding photographs. Mathematically, a blurry image $B$ can be formulated as

$$
B=K \otimes I+N,
$$

where $K$ is a non-negative blur kernel, whose support is small compared to the image size, $I$ is the latent sharp image, $N$ is noise, and $\otimes$ denotes convolution. Image deblurring is to solve the deconvolution of Eq. (1) [14, 16]. The blur kernel $K$ needs to be recovered simultaneously with the latent sharp image $I$ from the observed blurry image $B$.

Currently, the kernel $K$ is represented by a $2 \mathrm{D}$ matrix. But if carefully analyzing the process of photography, we can find that it actually consists of three aspects: trajec-

* This work was supported in part by Natural Science Foundation of China (NSFC) under contract No. 61303151, 61331015 and 61390514. tory, intensity and point spread function (PSF) ${ }^{1}$. Each of them would be able to clearly correspond to a property of the camera and scene system. Specifically, the trajectory describes the projection of camera shake in 2D image plane. Intensity reflects staying time of shaking camera and moving object in every position. PSF is decided by camera focus, scene depth and camera motion at the perpendicular direction of image plane.

Many deblurring approaches attempt to solve three aspects of the blur kernel $K$ simultaneously [4, 30, 22, 11, $18,25,13,17,12]$, which often results in local minimum in the deconvolution [16] and wrong blur kernel for extremely complicated camera motion. Visually, the deblurred results may contain ringing and other artifacts. To solve this problem, we consider the deconvolution in a brand new perspective with the idea of separable kernel. We propose decomposing a blur kernel into three individual descriptors (trajectory, intensity and PSF), which can be optimized separately by taking their physical properties as priors.

Before us, non-uniform deconvolution approaches have been proposed from spatial-variant kernels $[10,7,8,26]$ or motion-variant kernels [6, 23, 28, 24]. They actually consider multiple blur kernels for one blurry image. On the contrary, the proposed approach improves individual descriptor of one blur kernel. To demonstrate the advantage, we extract one-pixel-width trajectory of the blur kernel and introduce the continuous constraint on it. Following the idea of the evolutionary algorithm (EA) [5], we randomly change a local segment of the trajectory and perform the deconvolution with the change. The deblurred result is evaluated on the selected blocks with salient structures to decide whether the change is accepted or not.

Our experimental results show that the optimization on trajectory can significantly improve the deconvolution. Although earlier hardware-based work did consider the trajectory with the help of extra sensor [9], our work is the first paper that quantify the effect of the trajectory in the deconvolution without any extra hardware device. The pro-

\footnotetext{
${ }^{1}$ Unlike in [22], the PSF in our paper is not equal to the blur kernel. PSF is decided by camera focus, scene depth and camera motion at the perpendicular direction of image plane.
} 
posed separate kernel is also suitable to optimize other two parts of a blur kernel. But in this paper we will focus on the trajectory optimization hereafter. Our contributions are summarized as follows.

- We propose an innovative separable kernel concept to provide a more precise description of blurry effect.

- Our separable kernel is effective in respectively revealing the trajectory, intensity and point spread function characteristics of the kernel.

- We successfully show that the optimization of the trajectory will lead to a more accurate reconstruction of the kernel as well as the latent sharp image.

The outline of the rest of the paper is as follows. Section 2 introduces related researches. Section 3 presents our proposed separable kernel. Then in Section 4 we specifically propose an algorithm to optimize the trajectory individually. Section 5 presents experiment results and Section 6 concludes this paper.

\section{Related Work}

Uniform Deconvolution - It assumes one blur kernel for one blurry image. The researches in this line focus on introducing different regularization (priors) for better deconvolution. Fergus et al. introduce image statistical characteristics to solve the blur kernel [4]. Yuan et al. use a noisy but clear image as priors [30]. Shan et al. propose a unified probabilistic model [22]. Joshi et al. take color information as priors [11]. Cai et al. [1] recover latent image by enhancing the sparsity of blur kernels in the curvelet system. Li et al. work on the blurry video to generate a clear panorama [18]. $\mathrm{Xu}$ et al. exploit edges as priors [25]. Krishnan et al. propose the sparsity priors [13]. Levin et al. take the overall shape of the posterior distribution as priors [17]. The key problem in this line is that the blurry images are hard to be characterized by one blur kernel. No matter what priors are introduced, they may result in local minimum.

Spatial-variant Deconvotion - The researches in this line study the multiple blur kernels with different PSFs for one blurry image. Joshi et al. estimate PSFs via sharp edges [10]. Harmeling et al. propose to solve the devolution by partitioning the blurred images into multiple regions, where each region has its own kernel [7]. Hirsch et al. further extend the work to multiple frames [8]. Xu et al. estimate the PSFs with depth information [28]. When the blurry images have different scene depths, it is better to use multiple blur kernels with different PSFs.

Motion-variant Deconvolution - The researches in this line study different projections of camera motion in a scene. Gupta et al. propose modeling camera motion by motion density function [6]. Tai et al. model the camera motion by the projective motion path [23]. Xu et al. introduce simplified homograph blur model [28]. Whyte et al. propose modeling camera rotation motion as opposed to translation [24]. They mainly study a more general camera motion in 3D space and try to model the camera rotates, tilts, or moves along the camera axis during exposure. Our approach optimizes the trajectory in the 2D kernel plane and thus is more simple. Furthermore, the evolution process makes our approach easier to skip out of local minimum.

Partial Motion Deconvolution - The researches in this line study the blur caused by fast moving objects in scenes. Thus only partial of images is blurred. Levin et al. propose to detect the blurry regions by the statistic of derivative filters [15]. Liu et al. identify the blurry regions with the help of color, gradient and spectrum information [19]. Dai et al. propose a linear constraint similar to the optical flow field [3]. Cho et al. use the orthogonal parabolic exposures to keep high frequency image contents [2]. In this paper, we mainly focus on the blur caused by camera shake.

Hardware Assistant Deconvolution - It is usually tough to directly apply deconvolution on blurry images. Consequently, Ramesh et al. proposed encoding the movement of objects by fluttering the shutter [21]. Joshi et al. proposed exploiting inertial measurement sensor data to recover the true trajectory of the camera during exposure [9]. The key problem in this line is that they need specially designed camera and cannot be applied to real world blurry images directly. Our approach can find a better camera motion WITHOUT any special device, and can be directly implemented on real world blurry images.

\section{Proposed Separable Kernel}

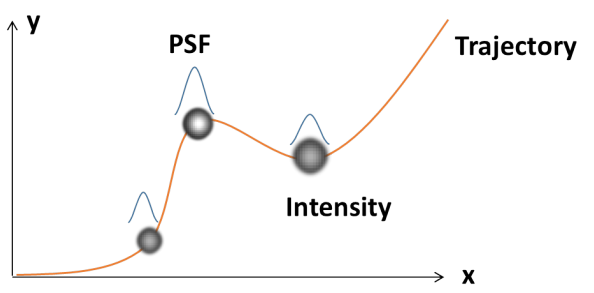

Figure 2. An exmple of our separable kernel.

Our proposed separable blur kernel is illustrated in Fig. 2 , where the orange line is the trajectory, the size of circle node represents the PSF, the brightness of the circle node indicates different intensities. Mathematically, let us denote $(x(t), y(t))$ to be a point at $t$-th moment that locates in the 2D image plane with horizontal and vertical axes being $x(t)$ and $y(t))$, respectively. Then the trajectory of the kernel during the moment $\left[0, t_{0}\right]$ can be represented by

$$
T=\int_{0}^{t_{0}} T(x(t), y(t)) d t
$$




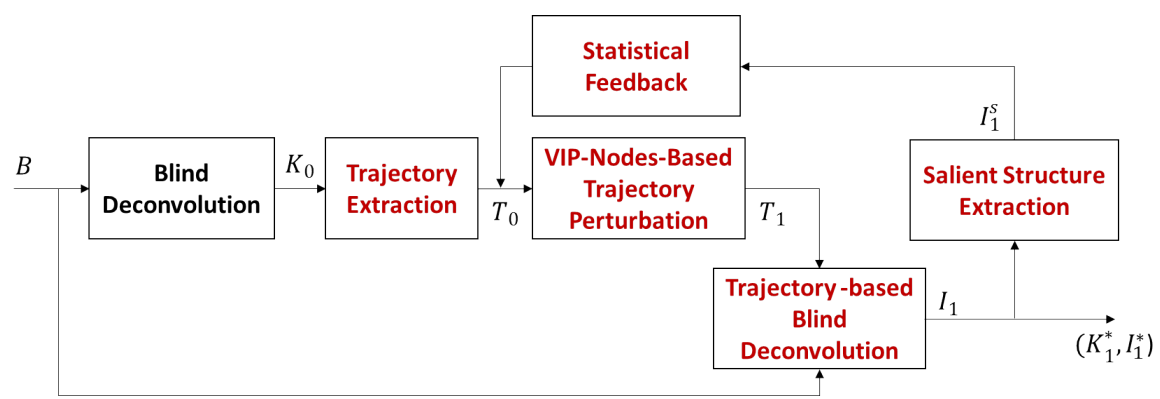

Figure 1. The dataflow of our proposed scheme for separable image deblurring.

where $T(x(t), y(t))=1$ indicates that the trajectory passes through $(x(t), y(t))$ in the $2 \mathrm{D}$ plane, vice versa. Then the separable kernel can be formulated as

$$
K=\int_{t} S(t) \times T(x(t), y(t)) \otimes G(x(t), y(t)),
$$

where $S(t)$ is the detention time in particular location of the 2D plane at $t$-th moment, which directly reflects the intensity value of one point in the kernel. $G(x(t), y(t))$ is the $2 \mathrm{D}$ Gaussian function at $t$-th moment. $\otimes$ represents the convolution operation.

- Obviously, our separable kernel can fully represent the traditional uniform kernel, with more specific and reasonable decomposition. One intrinsic constraint in our separable kernel is the continuity of the trajectory, which has not been well established in previous literature.

- As our separable kernel is effective in describing a general global model, such global model can then be mapped into different regions of one blurry image with different projections, leading to non-uniform kernel. In other words, our proposed separable kernel can be easily extended to a higher dimension to represent the non-uniform kernels.

Generally speaking, our separable kernel can be treated as a more concise description and observation for the camera and scene system beyond the uniform and non-uniform idea. The beauty of this separable concept is that our description of the real physical camera motion tends to be more realistic but succinct than traditional kernel representation.

\section{Deconvolution with Separate Trajectory}

To demonstrate the advantages of separable kernel, we propose an algorithm to optimize the trajectory of the blur kernel individually. As shown in Fig. 1, let us start with an observed blurry image $B$ followed by the blind deconvolution by existing approaches. A one-pixel-width trajectory $T_{0}$ of the blur kernel $K_{0}$ is first extracted. Inspired by the idea of evolutionary algorithm [5], we randomly change one segment of the trajectory. The changed trajectory $T_{1}$ is served as a regulation term in the trajectory-aided blind deconvolution. The deblurred image $I_{1}$ is statistically evaluated by selected salient blocks to decide if the changed trajectory $T_{1}$ is accepted or not. Fig. 1 gives a summary of the proposed scheme to validate our separable kernel idea.

\subsection{Trajectory Extraction}

Given the initial kernel $K_{0}$, we propose an iterative ordered region-growing process to extract the one-pixel-width trajectory from $K_{0}$. While region growing algorithms have been well studied before, we treat the problem in a completely different way by firstly representing the kernel as a energy-tree-structure.

Extracting energy-tree-structure from the kernel can be treated as producing a graph representation that describes the connectivity between all nodes in the kernel [29]. Specifically, at any given iteration, growth occurs from the node on the boundary of the grown region with the highest intensity,

$$
\begin{aligned}
& s_{k}=\max \left(B_{k}\right)=\left\{x \in B_{k} \mid \forall y \in B_{k}, I(x) \geq I(y)\right\}, \\
& \text { s.t. } G_{k}=\left\{s_{k}\right\}_{8} \backslash R_{k}, \\
& B_{k+1}=\left(B_{k} \cup G_{k}\right) \backslash s_{k}, \\
& R_{k+1}=R_{k} \cup G_{k}, \\
& B_{k} \subseteq R_{k} .
\end{aligned}
$$

where $s_{k}$ is the seed-point for $k$-th growing, $R_{k}$ represents the nodes within the region at $k$-th iteration, $B_{k}$ is the boundary of $R_{k}$ and $G_{k}$ is the set of growth nodes at the $k$ th iteration. $\{\cdot\}_{8}$ refers to the set of immediate 8-neighbors of a single node, and $\max \{\cdot\}$ denotes the node of maximum intensity of a set of nodes. A typical example for the extraction of energy-tree-structure from a kernel is depicted in Fig. 3(a) and 3(b).

Let $N_{s}$ and $N_{e}$ represent the starting and ending nodes of the trajectory, and let $P=\left(p_{1}, p_{2}, \cdots, p_{l}\right)$ be the trajectory where $l$ is the length, our goal is to have

$$
\begin{aligned}
& \operatorname{dist}\left(N_{e}, l\right)=\max _{P}\{f(P)\}, \\
& \text { s.t. } \quad f(P)=T\left(P_{\text {eff }} \wedge E\right)-\lambda T(P-E)
\end{aligned}
$$


where the objective function $f$ is formulated as the maximization of the possibility that the trajectory pass through most nodes of the energy-tree-structure. $E$ is the set of the bidirectional energy-tree-structure, i.e, $E(x, y)=1$ indicates that $x$ and $y$ has connection, and $E(x, y)=0$ indicates the disconnection. $T(\cdot)$ denotes the frequency (times) of one case occurs. $P_{e f f}$ is the effective trajectory regardless of the direction and duplication, i.e., once the connection appears, counting 1 for the frequency, otherwise counting 0 . The symbol $\wedge$ represents the "Logical Conjunction". Consequently, $T\left(P_{\text {eff }} \wedge E\right)$ is the frequency of the case that the effective trajectory passes through the energy-treestructure. Similarly, $T(P-E)$ is the frequency of the case that the trajectory never passes through the energytree-structure, given by

$$
T(P-E)=\sum_{i=1}^{l-1}\left[1-E\left(p_{i}, p_{i+1}\right)\right] .
$$

Under the recursive decomposition, $f(P)$ becomes

$$
\begin{aligned}
& f(P)=f\left(P^{\prime}, N_{e}\right) \\
& =\underbrace{f\left(p_{1}, p_{2}, \cdots, p_{l-1}\right)}_{f\left(P^{\prime}\right)}+\underbrace{\operatorname{Dup}\left(p_{l-1}, p_{l}\right)-\lambda E\left(p_{l-1}, p_{l}\right)}_{g\left(N_{e}^{\prime}, N_{e}\right)},
\end{aligned}
$$

where we denote $P^{\prime}=\left(p_{1}, p_{2}, \cdots, p_{l-1}\right), N_{e}^{\prime}$ be the ending point for $P^{\prime}$, and

$$
\begin{aligned}
& \operatorname{Dup}\left(p_{l-1}, p_{l}\right) \\
& =\left\{\begin{array}{cc}
0 & \left(p_{l-1}, p_{l}\right) \in\left\{\left(p_{1}, p_{2}\right),\left(p_{2}, p_{3}\right), \cdots,\left(p_{l-2}, p_{l-1}\right)\right\} \\
1 & \text { otherwise. }
\end{array}\right.
\end{aligned}
$$

Substituting (7) into (5),

$$
\operatorname{dist}\left(N_{e}, l\right)=\max _{P}\left\{f\left(P^{\prime}\right)+g\left(N_{e}^{\prime}, N_{e}\right)\right\} .
$$

Due to the fact that $N_{e}^{\prime}$ belongs to the 8-connected neighborhoods of $N_{e}$ (denoted as $N_{e}^{\prime} \in\left\{N_{e}\right\}_{8}$ ), and the length of the trajectory $P^{\prime}$ would be $l-1$, then (9) becomes,

$$
\operatorname{dist}\left(N_{e}, l\right)=\max _{N_{e}^{\prime} \in\left\{N_{e}\right\}_{8}}\left\{\operatorname{dist}\left(P^{\prime}, l-1\right)+g\left(N_{e}^{\prime}, N_{e}\right)\right\}
$$

Combining (7), (8) and (10), we can recursively find out the optimal solution of (5), i.e., given any starting node $N_{s}$, the optimal trajectory for arbitrary ending node $N_{e}$ and length $l$. Fig. 3 depicts a representative example of the trajectory extraction from the corresponding kernel.

\subsection{Random Trajectory Perturbation}

Examining $K_{0}$, it is interesting that several outstanding nodes (denoted as "VIP nodes" in this paper) have relatively higher intensity than the remaining nodes, as verified in Fig. 4 within the highlighted circles. Generally speaking, such

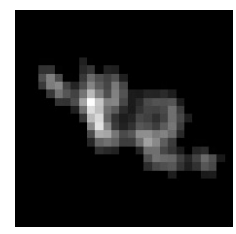

(a)

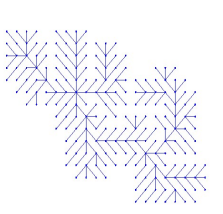

(b)

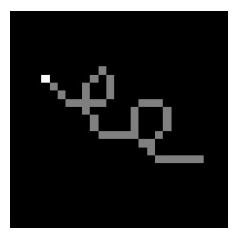

(c)
Figure 3. (a) initial kernel $\left(K_{0}\right)$; (b) energy-tree-structure of $K_{0}$; (c) extracted trajectory $T_{0}$.

VIP nodes may reflect the particular status that the camera motion stays for a relatively longer time. In other words, the VIP nodes tend to have high probability to be stable (unchanged or with minor changes), which allows us to allocate high trust on the VIP nodes in $K_{0}$. Consequently, during the random perturbation of the trajectory $T_{0}$, the VIP nodes will be approximated to be stable and $T_{0}$ will be decomposed into several sub-segments accordingly, as depicted in Fig. 4(c).

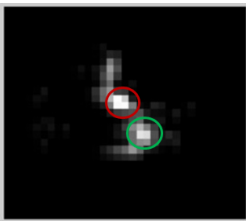

(a)

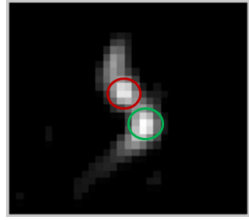

(b)

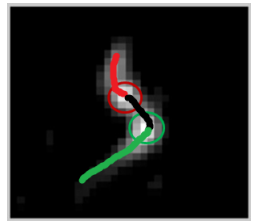

(c)
Figure 4. (a) VIP nodes in $K$; (b)VIP nodes in $K_{0}$; (c)Subtrace identified by VIP nodes.

Take any sub-segment as an example, we denote $S$ as the sequence of nodes in a sub-segment given by $\left\{\left(x_{1}, y_{1}\right),\left(x_{2}, y_{2}\right), \cdots,\left(x_{k}, y_{k}\right)\right\}, k$ is the number of nodes within sub-segment. Alternately, the gradients of nodes fully describe the relative directions among neighbor nodes. Such that $S$ can be represented with the sum of gradients,

$$
S=\sum_{i=1}^{k}\left(d x_{i}, d y_{i}\right)
$$

where $\left(d x_{i}, d y_{i}\right)$ are gradients of $i_{t h}$ node $\left(x_{i}, y_{i}\right)$ in subsegment. Then the random perturbation can be achieved simply by random switching the gradients within subsegment sequence,

$$
S^{\prime}=\underset{i \neq j}{R}\left(\left(d x_{i}, d y_{i}\right),\left(d x_{j}, d y_{j}\right)\right)
$$

where $R(\cdot)$ denotes the random switch operation.

\subsection{Trajectory-based Solution}

Recall that a general mathematical form of image blurring problem is Eq. (1). Under the same notation, we pro- 
pose a trajectory-based kernel optimization as follows

$$
\begin{array}{ll}
\min _{K, I} & \|\nabla B-\nabla I \otimes K\|_{2}^{2}+\lambda_{1} \frac{\|\nabla I\|_{1}}{\|\nabla I\|_{2}}+\lambda_{2}\|K\|_{1} \\
\text { s.t. } & \operatorname{Trace}(K)=T_{1},
\end{array}
$$

where $K, I, B$ are kernel, latent image, and blurry image respectively. $\frac{\|\nabla I\|_{1}}{\|\nabla I\|_{2}}$ is the latent image prior proposed in [13] to avoid delta kernel estimate. $\lambda_{2}|| K \|_{1}$ is the L1 norm serving as a sparse constraint. To solve Eq.(13) is non trivial because the trajectory is difficult to be represented with a straightforward function. Instead of dealing with the trajectory space itself, we propose to restrict the kernel space within a limited range of the trajectory by adding the different penalties (weights) on the kernel. Specifically, if the point is far from the trajectory, the penalty (weight) would be large, vice versa. On this basis, the optimization problem in Eq. (13) becomes

$$
\begin{array}{ll}
\min _{K, I} & \|\nabla B-\nabla I \otimes K\|_{2}^{2}+\lambda_{1} \frac{\|\nabla I\|_{1}}{\|\nabla I\|_{2}}+\lambda_{2}\|W \odot K\|_{1} \\
\text { s.t. } & W=1-E\left(T_{1}\right),
\end{array}
$$

where $W$ is the weight of $K, \odot$ is the Hadamard product and $E(\cdot)$ is the Gaussian mask. Given a trajectory, the corresponding $W$ would be a matrix with the value between $[0,1]$, as shown in Fig. 5. With the help of $W$ and a relatively large setting of $\lambda_{2}$, the shape of the kernel would be restricted to be alike $T_{1}$ in any possible way.

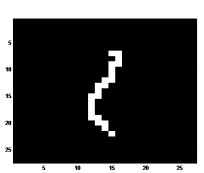

(a)

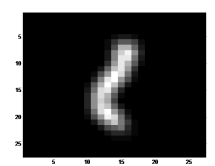

(b)

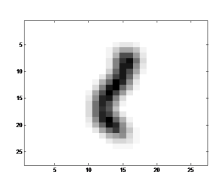

(c)
Figure 5. (a) Kernel initialization; (b) kernel initialization effects $(E)$; (c) weight $(W=1-E)$.

Considering the feedback for the random Perturbation from $T_{0}$ to $T_{1}$, since we have no reference for the evaluation of $T_{1}$ (or $I_{1}$ ), we introduce a penalty function by jointly considering "local smooth region smoothness (LM)" as well as the "local salient structure sharpness (LS)". Due to the fact that the ringing artifacts tend to appear in the smooth region that nearby a strong boundary, we extract plenty of salient structure patches (about 50 different patches around different strong boundaries), and compute the average of the LM and LS values respectively. Specifically, the the variance of the gradient in the smooth region nearby the strong boundaries is utilized to measure the smoothing level (indicating the level of ringing artifact), while the variance of the gradient in the boundaries is utilized to compute the sharpness (indicating the level of edge preservation) [20]. Under our definition, a smaller value of LM (indicates less ringing ar- tifacts in the salient structure) and a larger value of LS (indicates a sharper edge preserved in the salient structure) may lead to a better visual result.

\section{Experimental Results and Discussions}

We have performed experiments to verify the accuracy of the proposed scheme, where the tool in Jia etal. [27] is implemented as the initialization of kernel, and when kernel estimation finished, we use the same deconvolution tool to reconstruct the final latent image for all the concerned methods. Following the similar testing configuration as other BD papers, both the artificially generated blurry images and real world blurry images are used in experiments. In particular, we compare our method with Krishnan at al.[13] (2011) and Jia et al. [27] (2013) in the following experiments. Krishnan et al. propose the normalized sparsity priors for blind deconvolution. Jia et al. propose a robust deblurring software that incorporated the latest powerful blind deconvolution technologies [27] and [25] to remove camera shake.

As depicted in Fig. 6(a)-6(h), the shape of the kernel is well optimized in our kernel due to the proposed trajectory random perturbation and optimization. On the contrary, the estimated blur kernels in [13] and [27] fail to estimate the shape of the kernel, resulting in more ringing artifacts in the reconstructed image than our method (the roof and window of the house image).

Recall that one very important inherent constraint in our separable kernel is the continuity of the trajectory, indicating that our scheme is able to deal with the broken kernel. As demonstrated in Fig. 6(i)- 6(p), the broken kernel in Fig. 6(o) has been well optimized to be Fig. 6(p), leading to a visually superior reconstructed image (the shoulder and tripod of the cameraman image).

Similarly, due to the continuity constraint of the trajectory, our method is effective in suppressing the isolated noisy points or bifurcations that have negligible energy in the kernel, yielding better reconstructed results in both the kernel and latent image, as depicted in Fig. 6(q)-6(x) respectively.

We further testify the different performances of concerned methods using real world blurry image. Fig. 7(a) is the real blurry image where the two cropped text regions are highlighted with red and blue outlines and the green outline corresponds to the Fig. 7(e). Fig. 7(b) and 7(c) are the deblurred image using [13] and [27] respectively. Apparently, the zooming of the cropped text regions in their results are less readable with more ringing artifacts than our result. Examining the region highlighted with green outline, we can discover the rough structure of the blur kernel. Obviously, our estimated kernel is more accurate than the other two kernels, as shown in Fig. 7(f)-7(h).

To demonstrate that our scheme is robust to initial kernels, we take kernel estimated from [13] as initial for the 
following trajectory extraction and random perturbation. As depicted in Fig. 8, (a) is the real blurry image "flower" taken by mobile phone indoors, where the noise and saturation points are obvious. (b)(c)(d) are deburred results using [13], [27] and our scheme respectively. Although the visual quality of three deblurred results may appear similar at first glance, you may find that our kernel is accurate enough to recover the blurry line (as shown in Fig. 8(e)) to be a concentrated point.

\section{Conclusion and Future Work}

This paper proposes the concept of separable kernel for solving the deblurring problem. The blur kernel is decomposed into trajectory, intensity and PSF so that they can be optimized individually. To demonstrate the advantages of separable kernel, we also propose an algorithm to optimize the trajectory. Experimental results show that the proposed separable kernel and optimization can help the existing deblurring approach to get excellent results and correct blur kernels when camera motion is complicated.

The current trajectory is optimized by the proposed EAlike algorithm, which will lead to a high computation. In future, we can directly use the constraints on trajectory as priors. It will avoid the perturbations. In addition, this paper only shows the optimization on trajectory. Next, we will consider optimizing intensity and PSF individually. For example, for non-uniform deconvolution, we can first optimize the trajectory by using the whole blurred image and then optimize the multiple kernels with different PSFs but the same trajectory. The most advantage of separable kernel is to enable the strategy of divide-and-conquer in solving the deblurring problem.

\section{References}

[1] J.-F. Cai, H. Ji, C. Liu, and Z. Shen. Blind motion deblurring from a single image using sparse approximation. In Proc. of IEEE Conference on Computer Vision and Pattern Recognition (CVPR), 2009. 2

[2] T. S. Cho, A. Levin, F. Durand, and W. T. Freeman. Motion blur removal with orthogonal parabolic exposures. In ICCP, 2010. 2

[3] S. Dai and Y. Wu. Motion from blur. In CVPR, 2008. 2

[4] R. Fergus, B. Singh, A. Hertzmann, S. T. Roweis, and W. T. Freeman. Removing camera shake from a single photograph. In ACM Trans. on Graphics, 25(3):787-794, 2006. 1, 2

[5] D. B. Fogel. An introduction to simulated evolutionary optimization. Signal Neural Networks, IEEE Transactions on, 5(1):3-14, 1994. 1, 3

[6] A. Gupta, N. Joshi, C. L. Zitnick, M. Cohen, and B. Curless. Single image deblurring using motion density functions. In ECCV, 2010. 1, 2

[7] S. Harmeling, H. Michael, and B. Schölkopf. Spacevariant single-image blind deconvolution for removing cam- era shake. In Advances in Neural Information Processing Systems, pages 829-837, 2010. 1, 2

[8] M. Hirsch, S. Sra, B. Scholkopf, and S. Harmeling. Efficient filter flow for space-variant multiframe blind deconvolution. In $C V P R, 2010.1,2$

[9] N. Joshi, S. B. Kang, C. L. Zitnick, and R. Szeliski. Image deblurring using inertial measurement sensors. ACM Transactions on Graphics, 29(4):30, 2010. 1, 2

[10] N. Joshi, R. Szeliski, and D. J. Kriegman. Psf estimation using sharp edge prediction. In CVPR, 2008. 1, 2

[11] N. Joshi, C. L. Zitnick, R. Szeliski, and D. J. Kriegman. Image deblurring and denoising using color priors. In $C V P R$, 2009. 1, 2

[12] R. Köhler, M. Hirsch, B. Mohler, B. Schölkopf, and S. Harmeling. Recording and playback of camera shake: Benchmarking blind deconvolution with a real-world database. In $E C C V$, 2012. 1

[13] D. Krishnan, T. Tay, and R. Fergus. Blind deconvolution using a normalized sparsity measure. In $C V P R, 2011.1,2$, $5,6,7,8$

[14] D. Kundur and D. Hatzinakos. Blind image deconvolution. Signal Processing Magazine, IEEE, 13(3):43-64, 1996. 1

[15] A. Levin. Blind motion deblurring using image statistics. In NIPS, 2006. 2

[16] A. Levin, Y. Weiss, F. Durand, and W. T. Freeman. Understanding and evaluating blind deconvolution algorithms. In CVPR, 2009. 1

[17] A. Levin, Y. Weiss, F. Durand, and W. T. Freeman. Efficient marginal likelihood optimization in blind deconvolution. In CVPR, 2011. 1, 2

[18] Y. Li, S. B. Kang, N. Joshi, S. M. Seitz, and D. P. Huttenlocher. Generating sharp panoramas from motion-blurred videos. In CVPR, 2010. 1, 2

[19] R. Liu, Z. Li, and J. Jia. Image partial blur detection and classification. In $C V P R, 2008.2$

[20] R. Liu, Z. Li, and J. Jia. Image partial blur detection and classification. In CVPR, 2008. 5

[21] R. Raskar, A. Agrawal, and J. Tumblin. Coded exposure photography: motion deblurring using fluttered shutter. In ACM Trans. on Graphics, 25(3):795-804, 2006. 2

[22] Q. Shan, J. Jia, and A. Agarwala. High-quality motion deblurring from a single image. In ACM Trans. on Graphics (TOG), 27(3):73, 2008. 1, 2

[23] Y.-W. Tai, P. Tan, and M. S. Brown. Richardson-lucy deblurring for scenes under a projective motion path. Pattern Analysis and Machine Intelligence, IEEE Trans. on, 33(8):16031618, 2011. 1, 2

[24] O. Whyte, J. Sivic, A. Zisserman, and J. Ponce. Non-uniform deblurring for shaken images. International journal of computer vision, 98(2):168-186, 2012. 1, 2

[25] L. Xu and J. Jia. Two-phase kernel estimation for robust motion deblurring. In ECCV, 2010. 1, 2, 5

[26] L. Xu and J. Jia. Depth-aware motion deblurring. 2012. 1

[27] L. Xu, S. Zheng, and J. Jia. Unnatural 10 sparse representation for natural image deblurring. In $C V P R, 2013.5,6,7$, 8 


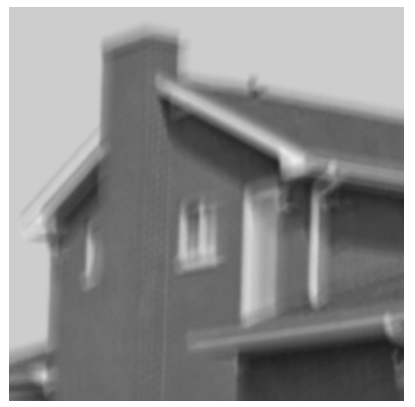

(a) Blurred House

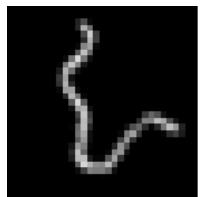

(e) Ground Truth Kernel

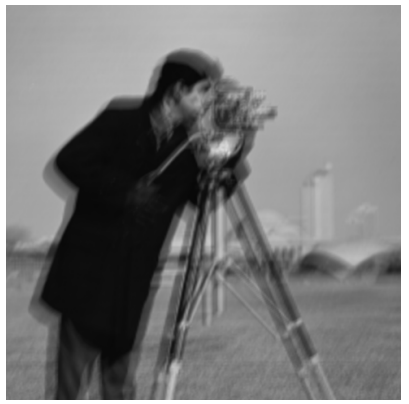

(i) Blurred Cameraman

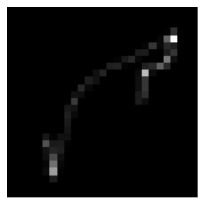

(m) Ground Truth Kernel

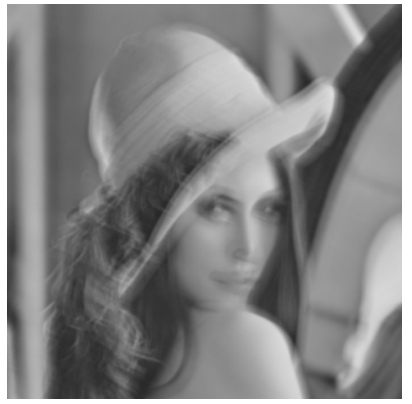

(q) Blurred Lena

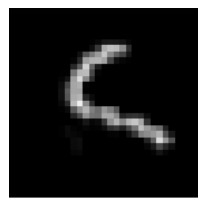

(u) Ground Truth Kernel

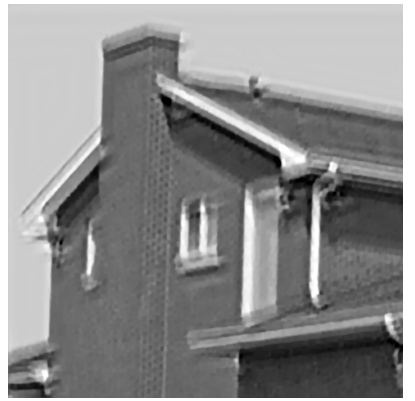

(b) [13]

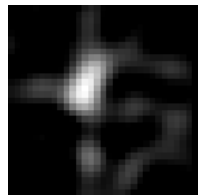

(f) Kernel of [13]

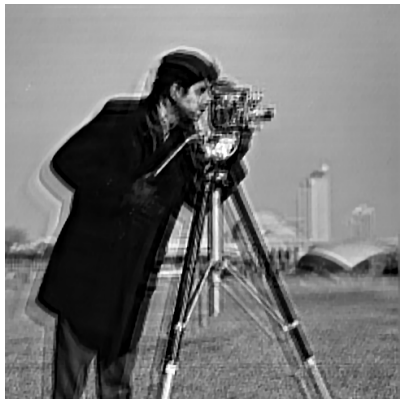

(j) [13]

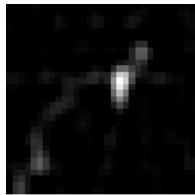

(n) Kernel of [13]

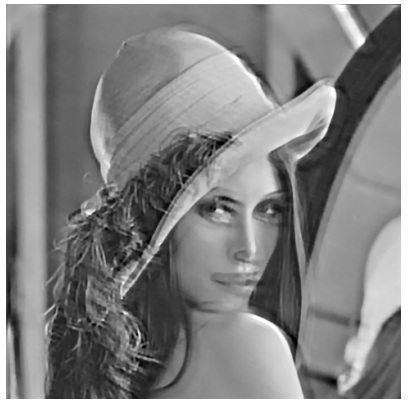

(r) [13]

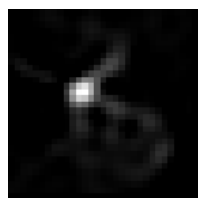

(v) Kernel of [13]
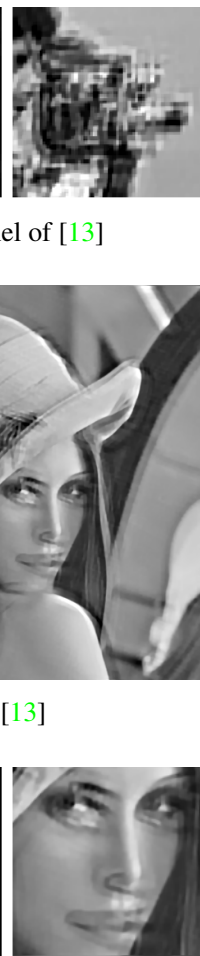

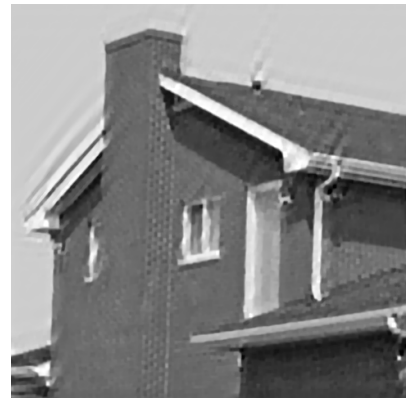

(c) [27]

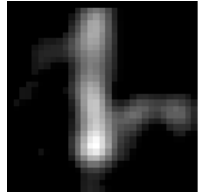

(g) Kernel of [27]

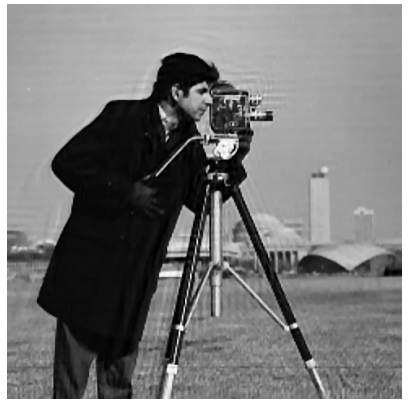

(k) [27]
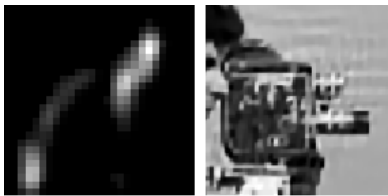

(o) Kernel of [27]

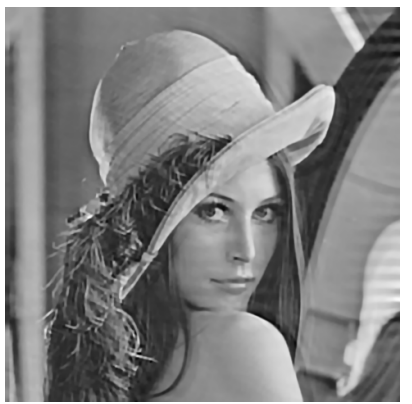

(s) [27]
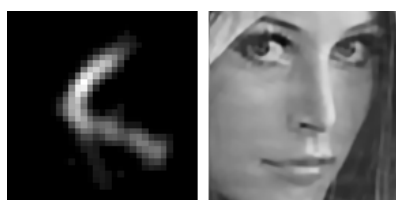

(w) Kernel of [27]

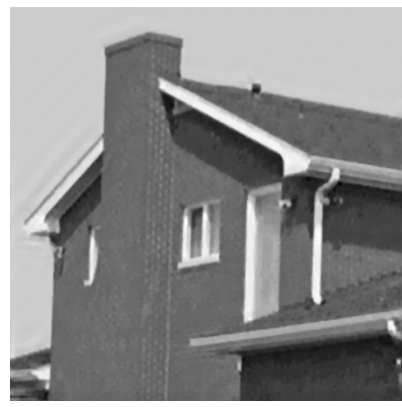

(d) Our Result
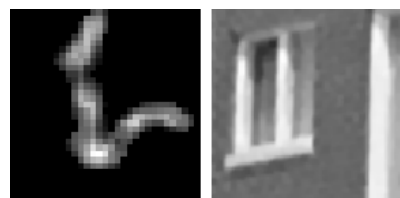

(h) Our Kernel

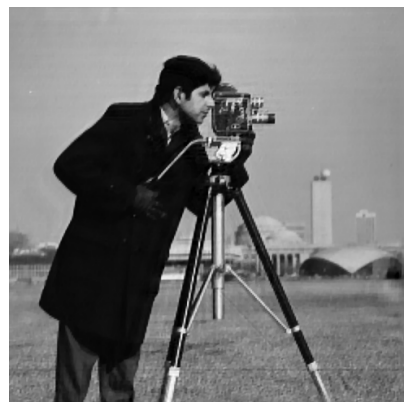

(1) Our Result

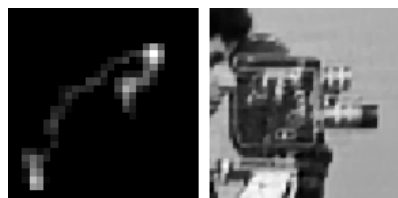

(p) Our Kernel

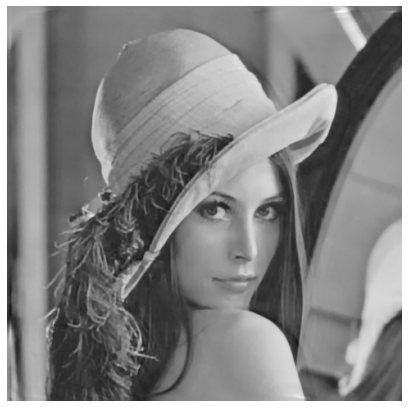

(t) Our Kernel

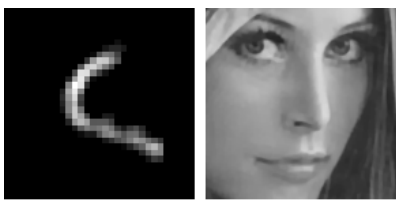

(x) Our Kernel

Figure 6. (a)(i)(q) Artificially blurred image; (e)(m)(u) ground truth kernel for (a)(i)(q); (b)(j)(r) deblurred image of [13]; (c)(k)(s) deblurred image of [27]; (d)(1)(t) our result. 

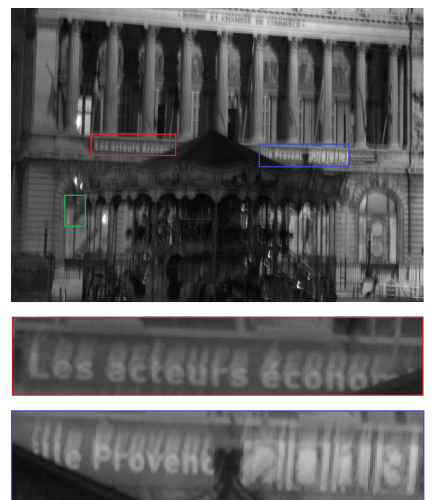

(a) Burry Image

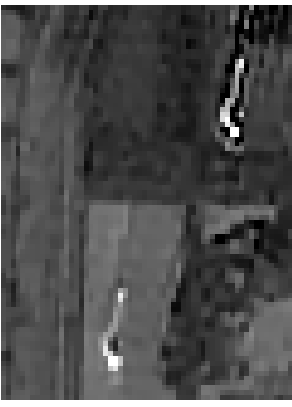

(e)
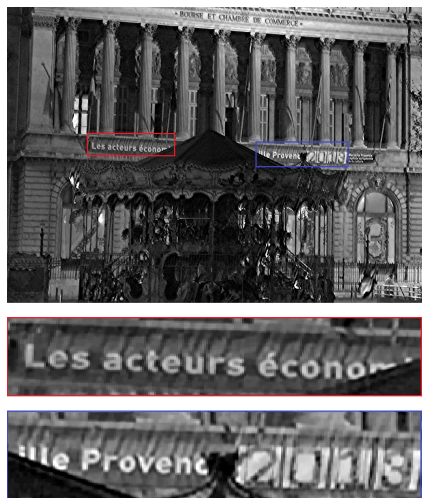

(b) [13]

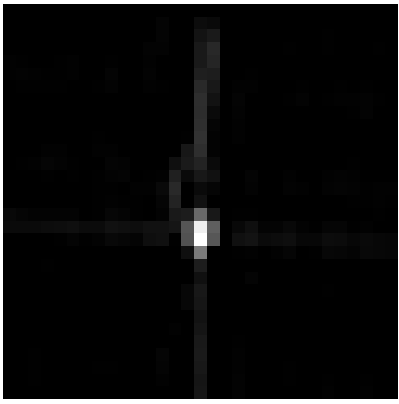

(f) Kernel of [13]
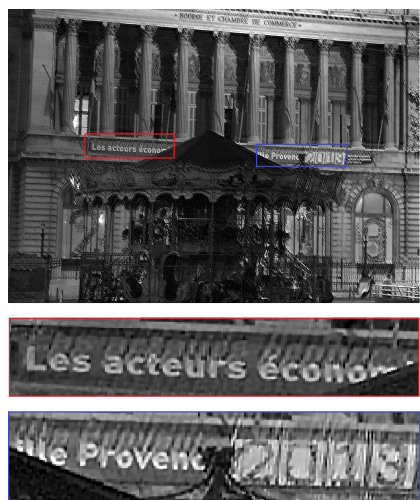

(c) [27]
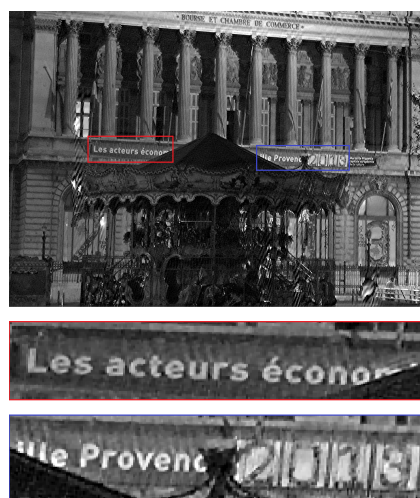

(d) Our Result

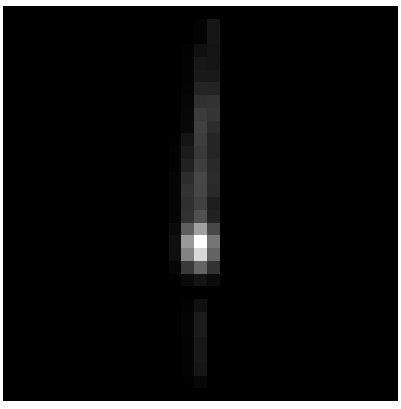

(g) Kernel of [27]

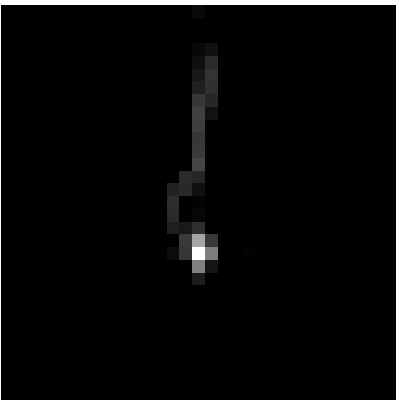

(h) Our Kernel

Figure 7. (a) Real blurry image; (b) deblurred image use [13]; (c) deblurred image use [27]; (d) our result; (e) cropped block from (a) (the green block); (f) blur kernel use [27]; (g) our blur kernel.

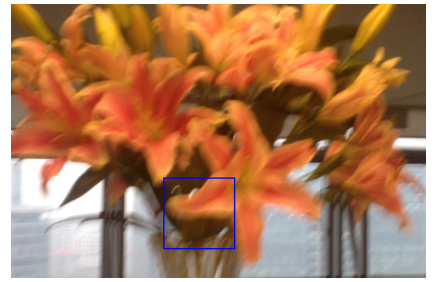

(a) Burry Image

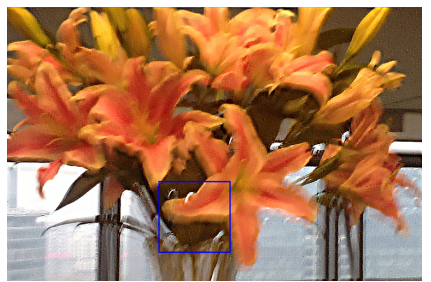

(b) [13]

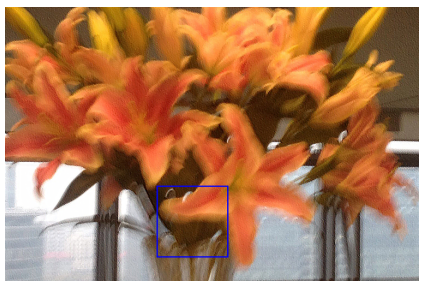

(c) $[27]$

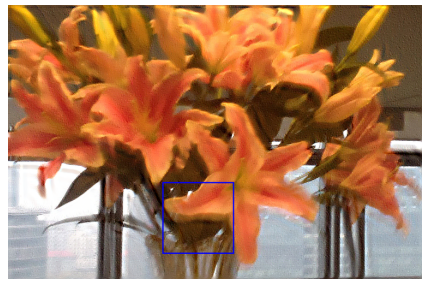

(d) Our Result

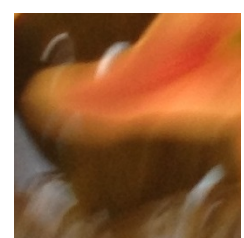

(e)

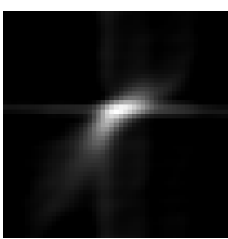

(f) Kernel of [13]
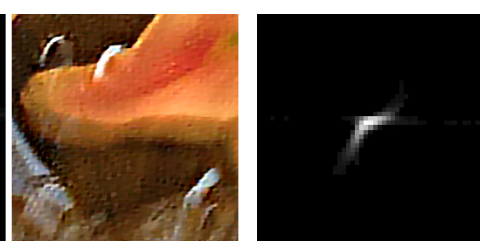

(g) Kernel of [27]
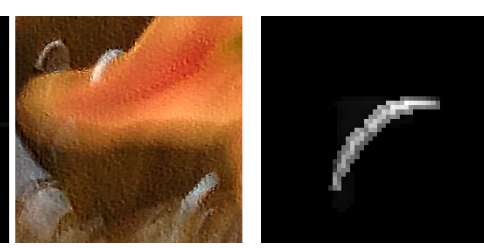

(h) Our Kernel

Figure 8. (a) Real blurry image; (b) deblurred image use [13]; (c) deblurred image use [27]; (d) our result; (e) cropped block from (a) (the green block); (f) blur kernel use [27]; (g) our blur kernel.

[28] Y. Xu, L. Wang, X. Hu, and S. Peng. Single-image blind deblurring for non-uniform camera-shake blur. Computer Vision-ACCV 2012, pages 336-348, 2012. 1, 2

[29] P. J. Yim, P. L. Choyke, and R. M. Summers. Gray-scale skeletonization of small vessels in magnetic resonance angiography. Medical Imaging, IEEE Trans. on, 19(6):568-
576, 2000. 3

[30] L. Yuan, J. Sun, L. Quan, and H. Y. Shum. Image deblurring with blurred or noisy image pairs. 26(3):1, 2007. 1, 2 